\begin{tabular}{|c|c|}
\hline Title & Numerical Simulation of Superconducting Coil W ound With No-Insulation NbTi Wire \\
\hline Author(s) & Noguchi, So; I toh, Ryusei; Hahn, Seungyong; Iwasa, Y ukikazu \\
\hline Citation & $\begin{array}{l}\text { IEEE Transactions on A pplied Superconductivity, 24(3), } 4900504 \\
\text { https://doi.org/10.1109/T A SC.2013.2287909 }\end{array}$ \\
\hline Issue Date & $2014-06$ \\
\hline Doc URL & http://hdl.handle.net/2115/56583 \\
\hline Rights & $\begin{array}{l}\text { (c) } 2014 \text { IEEE. Personal use of this material is permitted. Permission from IEEE must be obtained for all other uses, in } \\
\text { any current or future media, including reprinting/republishing this material for advertising or promotional purposes, } \\
\text { creating new collective works, for resale or redistribution to servers or lists, or reuse of any copyrighted component of } \\
\text { this work in other works. }\end{array}$ \\
\hline Tyре & article (author version) \\
\hline File Information & TA S24-3 Numerical Simulation.pdf \\
\hline
\end{tabular}

Instructions for use 


\title{
Numerical Simulation of Superconducting Coil Wound with No-Insulation NbTi Wire
}

\author{
So Noguchi, Member, IEEE, Ryusei Itoh, Student Member, IEEE, \\ Seungyong Hahn, and Yukikazu Iwasa
}

\begin{abstract}
A No-Insulation winding technique for an NbTi superconducting coil is expected to improve its dynamic stability during charging or discharging. Experimental results, recently reported, demonstrated the charging stability enhancement of an NI coil. In an NI winding, an amount of current can bypass to adjacent wires to avoid the generation of a local hot spot and, as a result, the dynamic stability is improved. In spite of the potential technical merits of the NI technique, the charging responses of an NI coil, including the non-uniform current distribution under a time-varying condition, have not been investigated analytically yet. In this paper, we propose a simulation method using an equivalent circuit equation for an NI coil with its inductance and the contact turn-to-turn resistances taken into consideration. The simulation results were analyzed in three aspects: terminal voltage, loss energy, and stored magnetic energy.
\end{abstract}

Index Terms-circuit equation, dynamic stability, noinsulation coil, superconducting magnet.

\section{INTRODUCTION}

$\mathrm{R}$ ECENTLY, it was reported that a No-Insulation (NI) technique improved the dynamic stability of an NI superconducting coil, both in LTS (low temperature superconductor) and HTS (high temperature superconductor). So far, the NI technique has been studied for applications of MRI (magnetic resonance imaging) magnet wound with LTS wires [2], high field HTS magnet [3], [4] and HTS racetrack coil for rotating machines [5], [6]. In LTS coils, mostly wound with $\mathrm{NbTi}$ and/or $\mathrm{Nb} 3 \mathrm{Sn}$, it is well known that wire motion often induces a premature quench during an initial charging. Insulation of the superconducting wire prevents a current from bypassing a local normal zone, created by the wire motion, to adjacent wires. Then the current in the local normal zone generates a Joule loss that may lead to the premature quench. On the other hands, as reported in [2], the current in an NI coil could bypass through the adjacent wires so that the Joule heating may be avoided, i.e., the dynamic stability of the coil may be improved. The NI coil, however, has some disadvantages. Firstly, charging an NI coil takes longer than its insulated counterpart due to the presence of the bypassing

Manuscript received July 16, 2013.

S. Noguchi and R. Itoh are with the Graduate School of Information Science and Technology, Hokkaido University, Kita 14 Nishi 9, Kita-ku, Sapporo 060-0814, Japan (phone: +81-11-706-7671; fax: +81-11-706-7670; e-mail: noguchi@ @si.ist.hokudai.ac.jp).

S. Hahn and Y. Iwasa are with the Francis Bitter Magnet Laboratory, Massachusetts Institutes of Technology, 170 Albany St., Cambridge, MA 02139, USA.
TABLE I

SPECIFICATIONS OF NI COIL WOUND WITH NI NbTi WIRE

\begin{tabular}{c|c}
\hline \hline Diameter of NbTi wire [mm] & 0.4 \\
Inner diameter $r_{1}[\mathrm{~mm}]$ & 101.6 \\
Outer diameter $r_{2}[\mathrm{~mm}]$ & 105.9 \\
Length $h[\mathrm{~mm}]$ & 11.2 \\
Number of solenoid layers & 6 \\
Number of total turns & 165 \\
Calculated inductance [mH] & 5.11 \\
\hline \hline
\end{tabular}

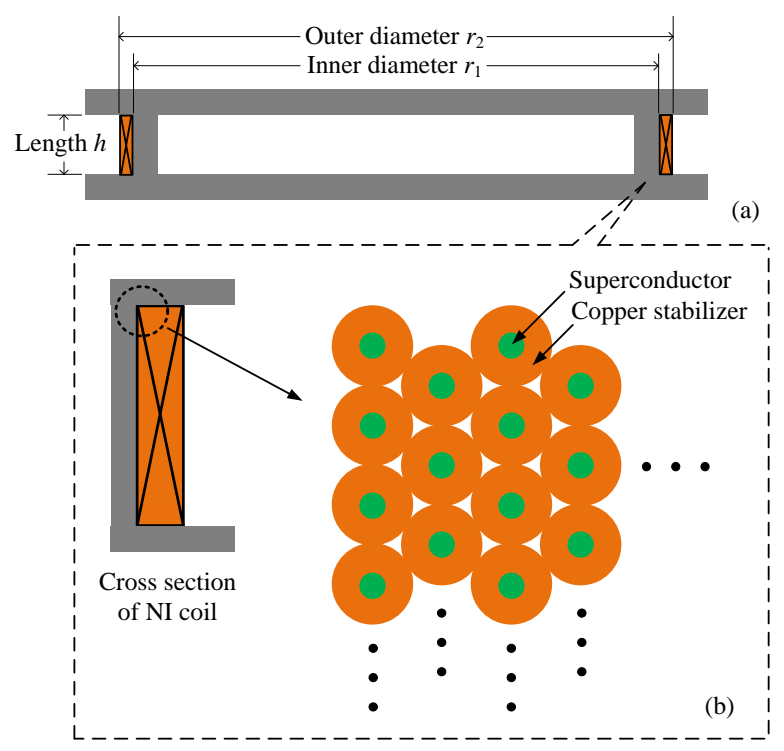

Fig. 1. The conceptual view of (a) the cross section of the NI coil and (b) the no-insulated $\mathrm{NbTi}$ wire structure.

current path in the NI coil. In addition, during charging or discharging, extra Joule loss is generated in the contact resistance [7]. Therefore, for the NI technique to become practically applicable to actual LTS magnets, the dynamic behavior of an NI coil needs to be investigated. Especially, a simulation technique, so far not thoroughly investigated, may be useful in estimation of the time-varying performance of an NI LTS coil and compromising the charging time and the Joule loss. This paper presents a simulation study on the dynamic performance of a no-insulation coil. The current flow path in an NI coil is investigated by use of an equivalent circuit equation. The proposed technique is also applied to simulate a partial-insulation (PI) coil that was recently proposed to reduce the charging delay and the consequent Joule loss of an NI coil [7]. The simulation results are compared with experimental ones for validation. 


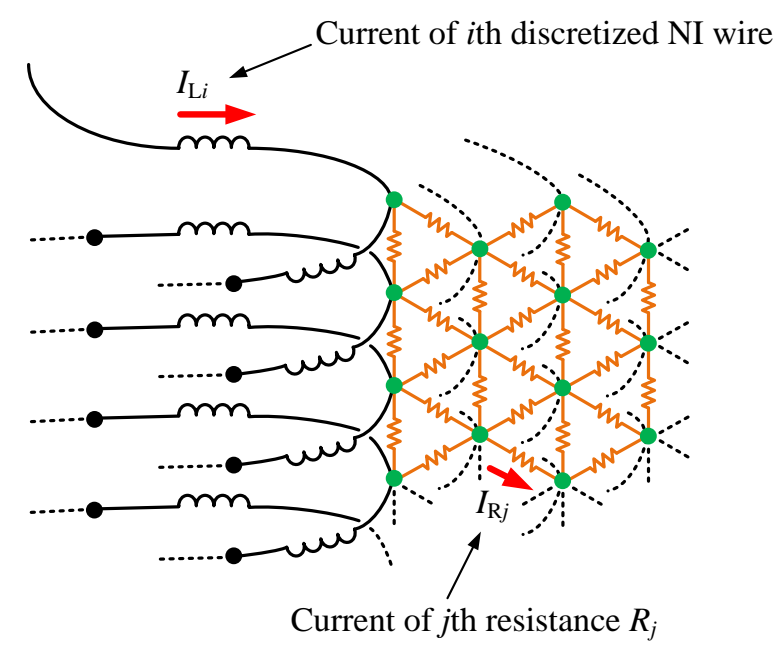

Fig. 2. The conceptual view of the equivalent circuit for an NI coil.

\section{SimUlATION APPROACH}

\section{A. Simulation Model}

Table I and Fig. 1(a) show the specifications and schematic views of an NI test coil wound with un-insulated (bare) NbTi wires. The wire consists of $\mathrm{NbTi}$ filaments and $\mathrm{Cu}$ stabilizer, of which a schematic cross-sectional view is shown in Fig. 1(b). Note that, in the NI wire view, no insulator, which in general surrounds the $\mathrm{Cu}$ stabilizer in a conventional $\mathrm{NbTi}$, is found so that a transport current can bypass through the turnto-turn contacts to avoid a local normal zone. With the inductance and resistance along the current path taken into consideration, an NI coil may be represented by a complex parallel circuit system.

\section{B. Simulation Method}

In the equivalent circuit, the NI wire is longitudinally divided into many short NI wire elements, and the discretized NI wires have a self-inductance and a mutual-inductance with each other. The resistance is composed by the resistance of the copper stabilizer and the contact between the adjacent wires in the equivalent circuit. Fig. 2 shows the schematic view of the equivalent circuit of the NI coil whose wire is longitudinally divided with the individual contact resistance.

The equivalent circuit equations can be derived from the Kirchhoff's law. The inflowing and outflowing currents are conditioned. The voltage of $i$ th discretized NI wire $V_{\mathrm{L} i}$ and the voltage of $j$ th contact resistances $V_{\mathrm{R} j}$ is given by

$V_{\mathrm{L} i}=\sum_{k=1}^{n_{\mathrm{L}}} M_{i k} \frac{\mathrm{d} I_{\mathrm{L} k}}{\mathrm{~d} t}$

$V_{\mathrm{R} j}=R_{j} I_{\mathrm{R} j}$

where $n_{\mathrm{L}}, M_{i k}, I_{\mathrm{L} k}, R_{j}$, and $I_{\mathrm{R} j}$ are, respectively, the number of the discretized NI wires, the mutual-inductance $\left(M_{i k}=L_{i}\right.$ when $i=k$, where $L_{i}$ is the self-inductance of the $i$ th discretized NI wire), the current flowing in the $k$ th discretized NI wire, the $j$ th resistance, and the current flowing in the $j$ th resistance. $R_{j}$

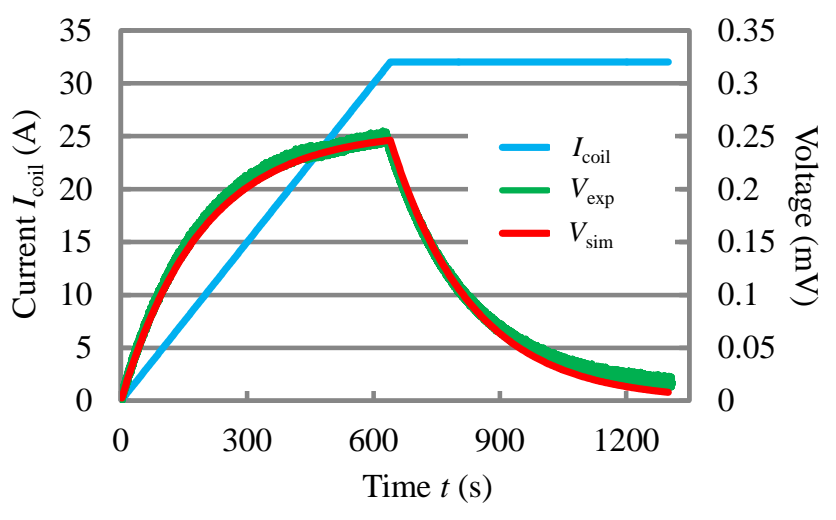

Fig. 3. Time vs. NI coil current $I_{\text {coil }}$ and terminal voltage. $V_{\text {exp }}$ and $V_{\text {sim }}$ show the terminal voltage, respectively experimental and simulation. The simulation results were obtained from the proposed method using the equivalent circuit.

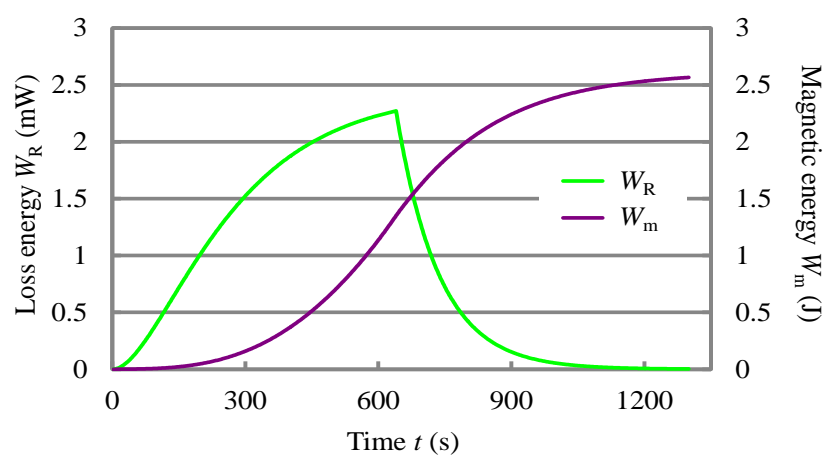

Fig. 4. Time transition of the loss energy $W_{\mathrm{R}}$ and the magnetic energy $W_{\mathrm{m}}$.

consists of the contact resistance and the resistance of the copper stabilizer; for estimation of the contact resistance, the contact length between the adjacent wires was considered with a constant line contact resistance of $0.013 \mathrm{~m} \Omega / \mathrm{m}$. All the resistances between the adjacent wires are assumed to be uniform in the simulation. In (1), the mutual-inductances $M_{i k}$ are calculated numerically based on the Biot-Savart law.

Using (1) and (2) with the calculated inductances, the following equivalent circuit equations are obtained:

$\sum_{l} V_{\mathrm{L} l}+\sum_{m} V_{\mathrm{R} m}=0$
$\sum_{p} I_{\mathrm{L} p}+\sum_{q} I_{\mathrm{R} q}=0$

where $l, m, p$, and $q$ are, respectively, the number of the inductances included in a closed circuit, the number of the resistances in the closed circuit, the number of the current flowing into the discretized NI wires connecting with a node, and the number of the current flowing into the resistances connecting with the node. (3) and (4) are built with the same number of the closed circuits and the nodes in the equivalent circuit. The system of the circuit equations is solved using an iterative solver. 


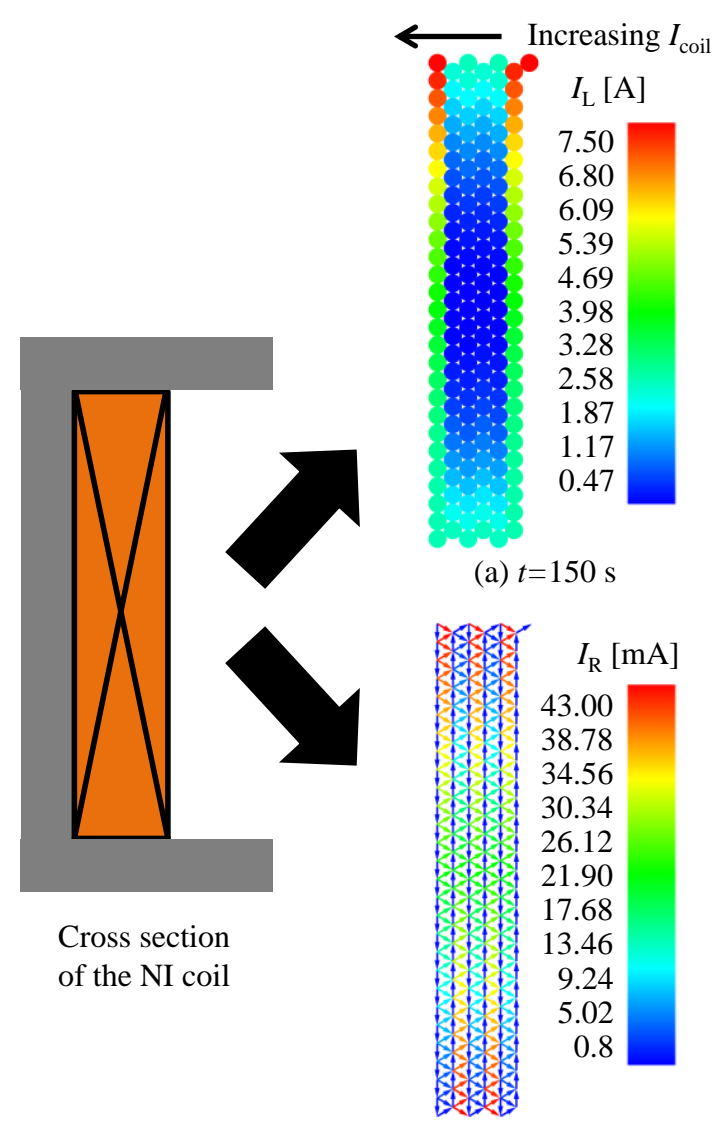

(f) $t=150 \mathrm{~s}$

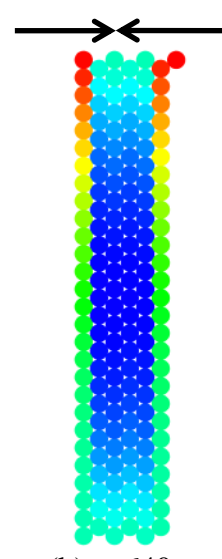

(b) $t=640 \mathrm{~s}$

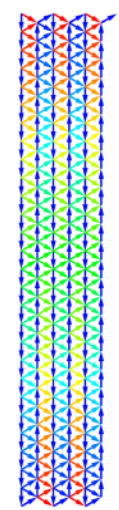

(g) $t=640 \mathrm{~s}$

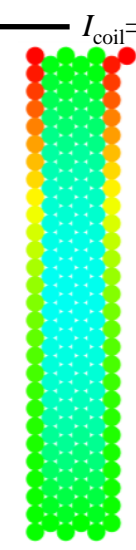

(c) $t=700 \mathrm{~s}$

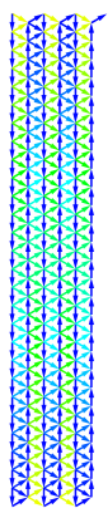

(h) $t=700 \mathrm{~s}$

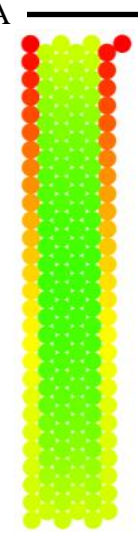

(d) $t=800 \mathrm{~s}$

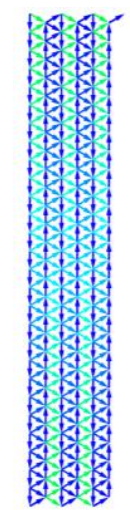

(i) $t=800 \mathrm{~s}$
$I_{\mathrm{L}}[\mathrm{A}]$

32.00

30.75

29.50

28.25

27.00

25.75

24.50

23.25

22.00

20.75

19.50

Fig. 5. Time transition of the current $I_{\mathrm{L}}$ which flows the discretized NI wire in the equivalent circuit at (a) $t=150 \mathrm{~s}$, (b) $t=640 \mathrm{~s}$, (c) $t=700 \mathrm{~s}$, (d) $t=800 \mathrm{~s}$, and (e) $t=1200 \mathrm{~s}$. The time transition of the current $I_{\mathrm{R}}$ which flows the resistance in the equivalent circuit at (f) $t=150 \mathrm{~s},(\mathrm{~g}) t=640 \mathrm{~s}$, (h) $t=700 \mathrm{~s}$, (i) $t=800 \mathrm{~s},(\mathrm{j}) t=1200 \mathrm{~s}$.
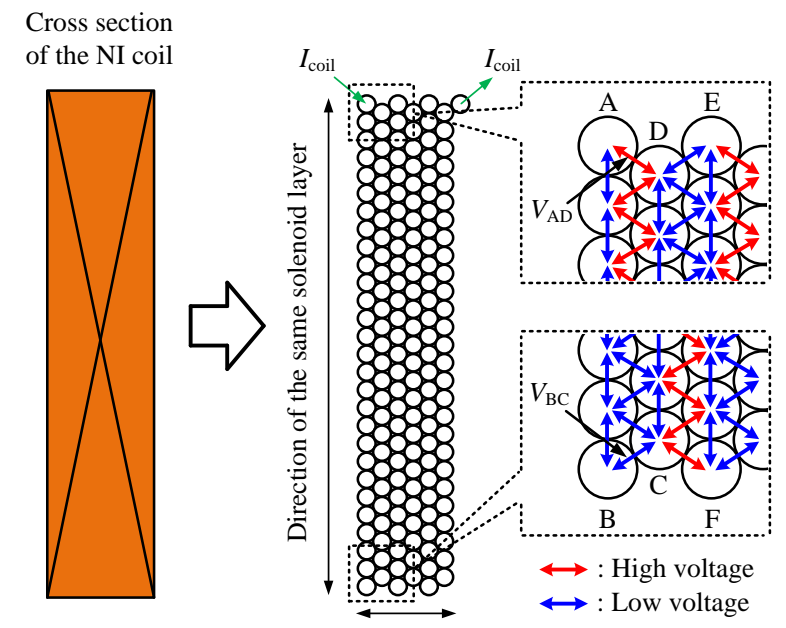

Direction of the different solenoid layer

Fig. 6. Enlarged view of the cross section of the NI coil.

\section{RESULTS AND DISCUSSIONS}

To investigate the transient behavior of an NI coil, the proposed simulation is performed and validated by comparison between the simulation and experimental results. In the simulation, the NI wire is circumferentially discretized into 4 segments in every turn.

\section{A. Terminal Voltage and Energy}

Fig. 3 shows the time vs. transition of the coil current and the terminal voltages, both experimental and calculated. The coil current $I_{\text {coil }}$ linearly increases to $32 \mathrm{~A}$ in $640 \mathrm{~s}$. After the coil current reaches $32 \mathrm{~A}$, it is kept to be constant. The ramping rate of the coil current is $0.05 \mathrm{~A} / \mathrm{s} . V_{\text {sim }}$ and $V_{\text {sim }}$ shows reasonably good agreement. The terminal voltages, both experimental and calculated, were approximately 0.25 $\mathrm{mV}$ when the coil current reaches its peak, $32 \mathrm{~A}$. Then, the terminal voltages decrease as the coil current was kept constant.

While the NI coil is charged, a portion of the coil current bypasses through the turn-to-turn resistive contacts, which generates Joule loss. This is one of the important side effects for NI coils, which needs to be carefully studied. The remaining portion of the coil current flows through the designated spiral path in the NbTi filament, which determines the magnetic energy stored in the NI coil. Fig. 4 shows time vs. Joule loss and stored magnetic energy graphs that were obtained from the simulated results by the proposed method. Initially, the loss energy increases with time and reaches to approximately $2.2 \mathrm{~mW}$ at $640 \mathrm{~s}\left(I_{\text {coil }}=32 \mathrm{~A}\right)$. The calculated total loss energy is $1.12 \mathrm{~J}$, which agrees well with the measured one, $1.23 \mathrm{~J}$. The stored magnetic energy is gradually 
increased as the "spiral" coil current increases; the peak stored energy is calculated $2.56 \mathrm{~J}$. With the coil self-inductance of $5.11 \mathrm{mH}$, the estimated stored magnetic energy is $2.62 \mathrm{~J}$. The good agreement between calculated and experimental results may validate the proposed simulation approach to descript the transient behavior of the NI coil using the equivalent circuit.

\section{B. Current Path}

In charging of an NI coil, the low Joule loss and the fast ramping, which are incompatible to each other, need to be compromised. For this purpose, we performed a simulation research to comprehend a non-uniform current path within the NI coil. Fig. 5 shows the temporal variation of the coil current distributions in the NbTi filaments of the NI coil, which were obtained using the proposed equivalent circuit approach. In Figs. 5(a) and (b), while the coil current $I_{\text {coil }}$ increases, most of the current flows in the innermost and outermost layers. After $I_{\text {coil }}$ reaches its peak $(32 \mathrm{~A})$, the distribution of the current $I_{\mathrm{L}}$ becomes more uniform as shown in Figs. 5(c)-(e). During charging the NI coil $(t<640 \mathrm{~s})$, the current in the top of the first inner layer bypasses to the second inner layer. Therefore, $I_{\mathrm{L}}$ decreases along the wire (axially downward in Fig. 5) in the first inner layer, i.e., a portion of the current bypasses to the adjacent wire as the current $I_{\mathrm{R}}$. Consequently, the current $I_{\mathrm{L}}$ in the center region is small, e.g., $0.47 \mathrm{~A}$ at $\mathrm{t}=150 \mathrm{~s}$ as shown in Fig. 5(a). The primary reason for the small current at and near the center region is the induced inverse voltage. Note that the currents flow mostly in the top and bottom of the coil during charging, $0<\mathrm{t}<640 \mathrm{~s}$.

Figs. 5(f)-(j) show the distributions of bypass current $I_{\mathrm{R}}$. In Figs. 5(f) and $(\mathrm{g})$, i.e. during charging $(\mathrm{t}<640 \mathrm{~s})$, the current $I_{\mathrm{R}}$ slowly increases as the coil current increases. After the coil current reaches $32 \mathrm{~A}$ and is kept constant, the current $I_{\mathrm{R}}$ gradually decreases and becomes zero at $\mathrm{t}=\sim 1200 \mathrm{~s}$.

The bypassing currents, large or small, between the layers on the top and bottom of the NI coil are shown in Fig. 5(f). The reason is that the high voltage between the different layers appears when its distance along the wire is long. The turns A and D in Fig. 6 are connected to each other, while the distance between A and D along the wire is "long". As the result, the voltage $V_{\mathrm{AD}}$ is high, and the current $I_{\mathrm{R}}$ is large there. On the other hand, the voltage $V_{\mathrm{BC}}$ between the turns $\mathrm{B}$ and $\mathrm{C}$ is very small since the distance along the wire is very short. Consequently, the currents of A-D and C-F are much larger than those of B-C and D-E.

From the above investigations, there are mainly two current paths between the terminals. One is the path completely along the NbTi filament of the wire and the other is the bypass on the top and bottom of the NI coil. The coil current flowing along the NbTi filament generates the magnetic energy, while the other one flowing in the resistive path is attenuated with generating the Joule loss. In view point of improvement of decreasing the loss energy and shortening the charging time, the partial insulation may be effective [7]. To investigate this issue, further researches are required.

\section{CONCLUSION}

The no-insulation (NI) technique may be useful for low temperature superconducting (LTS) magnet applications since it may be effective to improve the dynamic stability of an LTS coil. We investigated a numerical approach to estimate the transient behavior of an NI coil. In order to identify the loss energy of an NI coil during charging, an equivalent circuit model is proposed with the inductance of the discretized NI wire and the contact resistance between the adjacent wires taken into consideration.

The terminal voltage and the energy of the NI coil are computed by solving the equivalent circuit equations based on the Kirchhoff's laws. The simulation results agreed well with the experimental ones. Additionally, the non-linear current path within the NI winding is also investigated numerically. In simulation, the coil current is assumed to flow in two paths between the terminals. One is along the superconducting filament of the NI wire, and the other in the resistive path of the contacting wires. The former is associated with the stored magnetic energy, and the latter is attenuated with the loss energy.

In future, the proposed circuit analysis method is coupled with a thermal analysis. The dynamic stability for a heat has to be investigated by the analysis method considering the thermal behavior.

\section{REFERENCES}

[1] S. Hahn, D. K. Park, J. Bascuñán, and Y. Iwasa, "HTS pancake coils without turn-to-turn insulation," IEEE Trans. Appl. Supercond., vol. 21, no. 3, pp. 1592-1595, 2011.

[2] S. Hahn, D. K. Park, K. Kim, J. Bascuñán, and Y. Iwasa, "No-Insulation (NI) winding technique for premature-quench-free NbTi MRI magnet," IEEE Trans. Appl. Supercond., vol. 22, no. 3, p. 4501004, 2012.

[3] S. Choi, H. C. Jo, Y. J. Hwang, S. Hahn, and T. K. Ko, "A study on the no insulation winding method of the HTS coil," IEEE Trans. Appl. Supercond., vol. 22, no. 3, p. 4904004, 2012.

[4] S. Hahn, D. K. Park, J. Voccio, J. Bascuñán, and Y. Iwasa, "NoInsulation (NI) HTS inserts for > $1 \mathrm{GHz}$ LTS/HTS NMR magnets," IEEE Trans. Appl. Supercond., vol. 22, no. 3, p. 4302405, 2012.

[5] Y. Kim, S. Hahn, K. L. Kim, O. H. Kwon, H. Lee, "Investigation of HTS racetrack coil without turn-to-turn insulation for superconducting rotating machines," IEEE Trans. Appl. Supercond., vol. 22, no. 3, p. 5200604, 2012.

[6] H. Shin, K. L. Kim, Y. H. Choi, O. J. Kwon, S. Hahn, Y. Iwasa, H. G. Lee, "Effects of impregnating material on thermal and electrical stabilities of the HTS racetrack pancake coils without turn-to-turn insulation," IEEE Trans. Appl. Supercond., vol. 23, no. 3, p. 7700404, 2013.

[7] S. Noguchi, Y. Kim, S. Hahn, and Y. Iwasa, "Charging test of PartialInsulation (PI) winding technique for NbTi MRI magnets," presented at MT-23, 2013. 